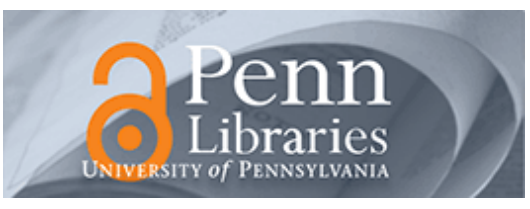

University of Pennsylvania

ScholarlyCommons

9-30-2016

\title{
Psychometric Testing of the Self-Care of Coronary Heart Disease Inventory (SC-CHDI)
}

\author{
Victoria V. Dickson \\ University of Pennsylvania \\ Christopher S. Lee \\ Karen S. Yehle \\ Ana Mola \\ Kenneth M. Faulkner
}

See next page for additional authors

Follow this and additional works at: https://repository.upenn.edu/nrs

Part of the Analytical, Diagnostic and Therapeutic Techniques and Equipment Commons, Behavioral Medicine Commons, Cardiology Commons, Cardiovascular Diseases Commons, Circulatory and Respiratory Physiology Commons, Medical Humanities Commons, Nursing Commons, and the Preventive Medicine Commons

\section{Recommended Citation}

Dickson, V. V., Lee, C. S., Yehle, K. S., Mola, A., Faulkner, K. M., \& Riegel, B. (2016). Psychometric Testing of the Self-Care of Coronary Heart Disease Inventory (SC-CHDI). Research in Nursing \& Health, 40 (1), 15-22. http://dx.doi.org/10.1002/nur.21755

This paper is posted at ScholarlyCommons. https://repository.upenn.edu/nrs/193

For more information, please contact repository@pobox.upenn.edu. 


\title{
Psychometric Testing of the Self-Care of Coronary Heart Disease Inventory (SC- CHDI)
}

\author{
Abstract \\ Although coronary heart disease (CHD) requires a significant amount of self-care, there are no \\ instruments available to measure self-care in this population. The purpose of this study was to test the \\ psychometric properties of the Self-Care of Coronary Heart Disease Inventory (SC-CHDI). Using the \\ Self-Care of Chronic Illness theory, we developed a 22-item measure of maintenance, management, and \\ confidence appropriate for persons with stable CHD and tested it in a convenience sample of 392 adults \\ (62\% male, mean age $61.4 \pm 9.6$ years). Factorial validity was tested with confirmatory factor analysis. \\ Convergent validity was tested with the Medical Outcomes Study MOS-SAS Specific Adherence Scale and \\ the Decision Making Competency Inventory (DMCI). Cronbach alpha and factor determinacy scores (FDS) \\ were calculated to assess reliability. Two multidimensional self-care scales were confirmed: self-care \\ maintenance included "consultative behaviors" (e.g., taking medicines as prescribed) and "autonomous \\ behaviors" (e.g., exercising 30 minute/day; FDS = .87). The multidimensional self-care management scale \\ included "early recognition and response" (e.g., recognizing symptoms) and "delayed response" (e.g., \\ taking an aspirin; FDS $=.76$ ). A unidimensional confidence factor captured confidence in each self-care \\ process $(\alpha=.84)$. All the self-care dimensions were associated with treatment adherence as measured \\ by the MOS-SAS. Only self-care maintenance and confidence were associated with decision-making \\ (DCMI). These findings support the conceptual basis of self-care in patients with CHD as a process of \\ maintenance that includes both consultative and autonomous behaviors, and management with symptom \\ awareness and response. The SC-CHDI confidence scale is promising as a measure of self-efficacy, an \\ important factor influencing self-care.
}

\section{Keywords}

coronary heart disease, self-care, adherence, measurement, instrument development

\section{Disciplines}

Analytical, Diagnostic and Therapeutic Techniques and Equipment | Behavioral Medicine | Cardiology | Cardiovascular Diseases | Circulatory and Respiratory Physiology | Medical Humanities | Medicine and Health Sciences | Nursing | Preventive Medicine

\section{Author(s)}

Victoria V. Dickson, Christopher S. Lee, Karen S. Yehle, Ana Mola, Kenneth M. Faulkner, and Barbara Riegel 
Psychometric Testing of the Self-Care of Coronary Heart Disease Inventory (SC-CHDI)

\author{
Victoria Vaughan Dickson $^{\mathrm{a}}$ \\ Associate Professor, New York University Rory Meyers College of Nursing \\ Christopher Lee \\ Carol A. Lindeman Distinguished Professor, Associate Professor, OHSU School of Nursing
}

Karen S. Yehle

Associate Professor, Purdue University School of Nursing, Center on Aging and the Life Course

Ana Mola

Director of Care Transitions and Population Health, NYU Langone Medical Center

Kenneth M. Faulkner

Doctoral Student, New York University Rory Meyers College of Nursing

Barbara Riegel, PhD, RN, FAHA, FAAN

Edith Clemmer Steinbright Professor of Gerontology, University of Pennsylvania, School of Nursing 
${ }^{\mathrm{a}}$ Corresponding Author:

Victoria Vaughan Dickson

Phone: 212-992-9426

Fax: 212-995-4564

Email:vdickson@nyu.edu

Address:

New York University

Rory Meyers College of Nursing

433 First Ave, \#742

New York, NY 10010

Conflict of Interest: None

Acknowledgements: Funded in part by NYU College of Nursing Pless Center Research Grant and the Center for Disease Control and Prevention (CDC) National Institute for Occupational Safety and Health (NIOSH) K01 Career Development Award in Occupational Safety and Health Research: 5K01OH009785-02

Keywords: Coronary heart disease, self-care, adherence, measurement, instrument development 


\begin{abstract}
Although coronary heart disease (CHD) requires a significant amount of self-care, there are no instruments available to measure self-care in this population. The purpose of this study was to test the psychometric properties of the Self-Care of Coronary Heart Disease Inventory (SC-CHDI). Using the Self-Care of Chronic Illness theory, we developed a 22 item measure of maintenance, management and confidence appropriate for persons with stable CHD and tested it in a convenience sample of 392 adults (62\% male, mean age $61.4 \pm 9.6$ years). Factorial validity was tested with confirmatory factor analysis. Convergent validity was tested with the Medical Outcomes Study Specific Adherence Scale (MOS-SAS) and the Decision Making Competency Inventory (DMCI). Cronbach's alpha and factor determinacy scores (FDS) were calculated to assess reliability. Two multidimensional self-care scales were confirmed: self-care maintenance included "consultative behaviors" (e.g., taking medicines as prescribed) and "autonomous behaviors" (e.g., exercising 30min/day) (FDS=0.87). The multidimensional self-care management scale included "early recognition and response" (e.g., recognizing symptoms) and “delayed response" (e.g., taking an aspirin) $(\mathrm{FDS}=0.76)$. A unidimensional confidence factor captured confidence in each self-care process $(\alpha=0.84)$. All the self-care dimensions were associated with treatment adherence as measured by the MOS-SAS. Only self-care maintenance and confidence were associated with decision-making (DCMI). These findings support the conceptual basis of self-care in patients with CHD as a process of maintenance that includes both consultative and autonomous behaviors, and management with symptom awareness and response. The SC-CHDI confidence scale is promising as a measure of self-efficacy, an important factor influencing self-care.
\end{abstract}

Keywords: Coronary heart disease, self-care, adherence, measurement, instrument development 


\section{Introduction}

Worldwide, coronary heart disease (CHD) is a leading cause of increased morbidity and mortality (Gaziano, 2007). In the United States, 1 in 3 adults has CHD (Mozaffarian et al., 2016). Advances in medical and surgical management of CHD have improved many patient outcomes including reduced cardiac mortality rates. However, individuals with $\mathrm{CHD}$ are at increased risk for unstable angina, myocardial infarction, and heart failure (Mozaffarian et al., 2016). Self-care is an essential component of the daily management of $\mathrm{CHD}$, with the need to practice behaviors that maintain stability (e.g., adherence to medication, dietary and exercise regimens), symptom monitoring and management. Unfortunately, there are no instruments available to measure selfcare in this population.

According to the middle range theory of self-care of chronic illness, self-care is "a naturalistic decision making process of maintaining health through health promoting practices and managing illness" (Riegel, Jaarsma, \& Stromberg, 2012, p.195). Self-care is said to encompass maintenance, monitoring, and management behaviors. Once an individual develops CHD, s/he needs to adhere to recommendations for diet, exercise, medication administration to maintain physiologic stability. Symptoms are common, but monitoring for them and managing them early can avoid emergency care and hospitalization (Wechkunanukul, Grantham, \& Clark, 2016).

Efforts to improve self-care are based on evidence that doing so will improve health outcomes. A recent systematic review of nurse-led interventions targeting self-care among patients with CHD found that the most frequently reported outcomes were improved quality of life and reduced healthcare utilization (Dickson et al., 2013). Of the 35 studies evaluated, few other than HF studies included a measure of self-care. In fact, measurement of self-care was fragmented into a specific behavioral adherence measurement (e.g., exercise or medication 
taking)(Gehi, Haas, Pipkin, \& Whooley, 2005; Toft et al., 2007) or acute symptom delay (Moser et al., 2006). The lack of an instrument to measure self-care among CHD patients is a significant gap in the literature and precludes scientists from linking an intervention to desired outcomes in many cases (Chodosh et al., 2005; Dickson et al., 2013; Hisashige, 2013).

Using clinical guidelines and standards of care for individuals with CHD (Fraker et al., 2007; Gibbons et al., 2003), we developed an instrument measuring self-care as defined by the middle range theory of self-care of chronic illness (Riegel et al., 2012). Instrument development and content validity are defined in detail below. The purpose of this study was to test the psychometric properties of the Self-Care of Coronary Heart Disease Inventory (SC-CHDI).

\section{Instrument Development}

Items on the SC-CHDI were designed to reflect the theoretical constructs of self-care maintenance and management. Self-care maintenance refers to behaviors used by individuals with a chronic illness to maintain stability of physical and mental health (Riegel et al., 2012). Self-care maintenance behaviors include recommendations from a health care provider as well as autonomous health promoting practices. Self-care management behaviors address evaluating changes in signs and symptoms and determining if action is needed. Self-care management also includes attention to treatment effectiveness and evaluation as to whether that self-care management approach should be used in the future. An additional scale, self-care confidence, which serves as a measure of self-care self-efficacy (Eller, Lev, Yuan, \& Watkins, 2016) reflects ability to perform each component of the self-care process. Self-care self-efficacy, measured with this scale in adults with heart failure, has been shown to be important in predicting successful self-care (Buck et al., 2015; Vellone et al., 2015).

Items on the self-care maintenance scale reflect 10 common behaviors recommended to 
maintain stability for persons with CHD: keep medical appointments, take aspirin or other blood thinner, check blood pressure, exercise, take medications, eat a low fat diet, use a medication reminder system, eat fruits and vegetables, avoid cigarettes and smokers, control body weight. These specific items were drawn from the American Heart Association's Life's Simple 7 and clinical guidelines for CHD. There is compelling evidence for the effectiveness of these behaviors in secondary prevention and risk reduction (Maddox \& Ho, 2009; Smith et al., 2011). For example, among patients with CHD, adherence to secondary prevention medications (e.g., antiplatelet agents, statins, beta-blockers) is associated with reduced morbidity and mortality (Fraker et al., 2007). Similarly, according to the 2011 American Heart Association guidelines, regular 30 to 60 minutes of moderate-intensity aerobic activity, such as brisk walking, supplemented by an increase in general physical activity can improve cardiorespiratory fitness and reduce risk (Smith et al., 2011). Respondents rate how frequently they engage in each behavior on an ordinal scale ranging from 1 (never or rarely) to 4 (always or daily). A 4-point scale was used to avoid having a neutral answer.

Items on the self-care management scale incorporate the concepts of monitoring and management with questions about symptom recognition and actions in response to symptoms (i.e., slow down, rest, take nitroglycerin if prescribed, call a provider for guidance, take an aspirin) and evaluation of the effectiveness of an action for use in the future. The self-care management scale is completed and scored if symptoms of chest pain, chest pressure, burning, heaviness, shortness of breath, or fatigue were experienced in the prior month. That is, if the respondent indicates that there were no symptoms in the prior month, the remainder of the scale is not completed or scored. Recognition is rated on an ordinal scale ( 0 not recognized to 4 very quickly). Management behaviors are rated in terms of likelihood of taking action ("how likely 
are you to") in response to a symptom (1 not likely to 4 very likely). Treatment effectiveness is judged with a single item asking surety of the helpfulness of the action, rated on an ordinal scale (0 I did not try anything, 1 not sure to 4 very sure).

The self-care confidence scale assesses confidence in the ability to stay free of symptoms, follow treatment advice, recognize health changes, evaluate the importance of symptoms, do something to relieve symptoms, and evaluate treatment effectiveness. As such, this scale reflects confidence in the ability to effectively perform all elements of self-care maintenance, monitoring and management. The self-care confidence scale is an ordinal scale ranging from 1 (not confident) to 4 (very confident).

Each of the two self-care scales reflecting maintenance and management is scored separately and standardized to 100 with higher scores indicating better self-care. The companion self-care confidence scale is also standardized 0-100; higher score indicate better self-care selfefficacy. That is, three separate scores are produced. For this reason, the SC-CHDI is called an inventory rather than an index because the term index suggests that separate scores are aggregated into a single number,(McDowell, 2006) which is not the case. The SC-CHDI is freely available on our website: http://www.self-careofheartfailureindex.com/.

\section{Methods}

\section{Content Validity}

Content validity is the degree to which an instrument has included appropriate items for the construct being measured. There are distinct phases of content validity: 1) careful conceptualization and domain analysis prior to item generation, and 2) evaluation of the relevant content through expert assessment(Polit \& Beck, 2006). In this study, conceptualization was based on the theory underlying the concept. 
Content validity was quantified using the Content Validity Index (CVI). We calculated the CVI for each item and then the entire instrument using the methods recommended by Lynn (1986). The CVI for each item was calculated as the proportion of experts who rated its content as relevant (3 or 4). Then CVI for the full instrument was calculated as the proportion of items judges rated as content relevant. With five or fewer judges, the item level CVI should be 1.00 and the scale level CVI should be $\geq .90$ (Polit \& Beck, 2006).

In this study, relevance of proposed content was judged by an expert panel of five cardiovascular nurse clinicians and scientists who were asked to rate the content relevance of each item on a scale of 1 (irrelevant) to 4 (extremely relevant) (Lynn, 1986). Items needing revision and comments about missing content were requested (Polit, Beck, \& Owen, 2007). After deleting two items from the maintenance scale that were judged as irrelevant by the panel of experts, the item level CVI was 1.00 and the scale level CVI was 1.00. The final self-care maintenance scale consists of 10 items. There are 6 items on the self-care management scale. The item level CVI of this scale was 1.00 and the scale level CVI was 1.00. Minor editing suggested by the expert panel was incorporated into this scale before we began psychometric testing. The item level CVI for the self-care confidence scale was 1.00 and the scale level CVI was 1.00. There are 6 items on the self-care confidence scale.

\section{Sample}

A convenience sample of 392 adults with CHD (e.g., history of myocardial infarction, angina) was used to assess the psychometric properties of the inventory. Individuals were recruited directly from outpatient clinical settings and cardiac rehabilitation programs from two large urban medical centers in the Northeastern and Midwestern U.S. and using ResearchMatch.org. This national electronic, web-based registry has a large population of volunteers who have consented 
to be contacted by researchers about health studies. ResearchMatch.org is supported by the U.S. National Institutes of Health as part of the Clinical Translational Science Award (CTSA) program. Participants were eligible to participate if they were over age 18 and reported having CHD (including angina, myocardial infarction, history of stent placement or coronary artery bypass graft surgery). Review and approval for this study was obtained from each of the study site’s Institutional Review Boards.

Participants completed: 1) a short demographic questionnaire, 2) the SC-CHDI, 3) the Medical Outcomes Study Specific Adherence Scale (MOS-SAS), and 4) the Decision Making Competency Inventory (DMCI). These instruments were used in validity testing because self-care maintenance involves behaviors that maintain stability including those assessed by the MOSSAS and self-care is defined as a decision-making process.

The MOS-SAS is a valid and reliable instrument (Coefficient $\alpha$ is .78; test-retest correlation .55) that has been widely used in medical outcomes research including CHD research (Huffman et al., 2015; Wu, Moser, Chung, \& Lennie, 2008) to assess adherence to eight commonly prescribed behaviors: follow a low salt diet, follow low fat diet, monitor weight, take prescribed medications, cut down or stop smoking, avoid alcohol, exercise regularly and monitor symptoms (Hays et al., 1994). Participants rate how often they have done each item over the past month using a 5-point Likert scale (0-none of the time to 5-all of the time). Scores are summed and transformed to $0-100$ score to form a single scale score.

The Decision Making Competency Inventory (DMCI) (Coefficient $\alpha=.86$ ) is a 20-item measure of decision making skill (Miller \& Byrnes, 2001). Participants rate themselves on how they make decisions, for example: "I think about similar past decisions I made and what happened" using a 5-point scale (1- not at all like me to 6-very much like me). The DMCI has 
four domains: 1) informed awareness (i.e., being reflective in the decision-making process and gathering adequate information in order to make an informed decision); 2) self-appraisal (being mindful of personal qualities that can affect the consequences of choices; 3) autonomy in critically evaluating options and making a choice; and 4) confidence in making appropriate decision (Miller \& Byrnes, 2001). The DCMI total score, and informed awareness and selfappraisal subscales were used in this analysis because these processes are specified in the theory of self-care of chronic illness as influencing self-care.

\section{Analysis}

Descriptive statistics of proportions, central tendency and dispersion were used to describe the sample. SC-CHDI item response proportions, means and standard deviations, and item-test correlations (i.e. the correlation between the item and a scale formed by all other items in that domain) were quantified using Stata v14 (College Station, TX). These data provide evidence of item distribution as well as potential poor item fit. The SC-CHDI is a new measure but was based on extensive prior study in related cardiovascular patient populations and published theory; hence, confirmatory factor analysis was performed using Mplus v.7 (Los Angeles, California) to test factorial validity. Weighted least square parameter estimation with mean- and variance-adjusted statistics was chosen given the ordinal nature of item responses (Flora \& Curran, 2004). To assess model fit, overall model $\chi^{2}$ tests (non-significant), comparative fit indices (CFI) (>0.95), Tucker-Lewis indices (TLI) (>0.95), root mean square errors of approximation (RMSEA) $(<0.08)$, and Standardized Root Mean Square Residual (SRMSR) $(<0.08$ acceptable) were calculated using common thresholds of acceptable fit (Hu \& Bentler, 1999; Schnermelleh-Engel, Moosbrugger, \& Muller, 2003; Yu, 2002). Convergent validity was tested with both adherence (MOS-SAS) and decision-making (DMCI) using linear 
correlations (with significance $\mathrm{p}<0.05$ as the index of convergence).

Cronbach's alpha and factor determinacy scores (FDS; $\geq 0.70$ acceptable, range $0-1$ (Brown, 2003; Tabachnick \& Fidell, 2013) were calculated to assess reliability for unidimensional and multidimensional scales respectively.

\section{Results}

The sample of 392 adults was mostly male (62\%) and mostly Caucasian (71\%). The mean age was $61.4 \pm 9.6$ years; mean duration of $\mathrm{CHD}$ was $6 \pm 8$ years. A mixture of patients with stable CHD (e.g., angina, prior myocardial infarction, history of stent placement or coronary artery bypass graft surgery) was enrolled. Further details describing the sample are shown in Table 1 . Validity and reliability analysis for each scale is described below.

\section{Self-Care Maintenance Scale}

\section{Factor Analysis, Convergent Validity, and Reliability}

A 2-factor multidimensional structure to the maintenance items had a marginal fit $(\chi 2=124.6, \mathrm{p}<0.001 ; \mathrm{RMSEA}=0.088 ; \mathrm{CFI}=0.904, \mathrm{TLI}=0.833, \mathrm{SRMR}=0.065)$ (Figure 1 and Table 2). A self-care maintenance factor named "consultative behaviors" included six items (i.e. \#1 keeping appointments, \#2 taking aspirin, \#3 taking other medicines as prescribed, \#5 checking blood pressure, \#7 using a medication reminder system, and \#9 avoiding smoking and smokers). Another factor was named "autonomous behaviors", which included four items (i.e. \#4 exercising 30min/day, \#6 asking for low sodium foods, \#8 eating fruits and vegetables, and \#10 weight maintenance). These dimension names reflect the theoretical definition of self-care as a process that may be entirely autonomous or may reflect recommendations from a health care provider (Riegel et al., 2012). Item-test correlations for this scale ranged from .44 to .62 with no redundancy or low correlation was found. 
Convergent validity analysis showed that both self-care maintenance factors were associated significantly with adherence $(\mathrm{P}<001)$ and with decision making. The autonomous factor was significantly associated with all three of the DCMI domains at $\mathrm{P}<.001$. The consultative factor was associated with only the total DCMI score $(\mathrm{r}=.228, \mathrm{P}=.003)$ and selfappraisal domain $(\mathrm{r}=.271, \mathrm{P}<.001)$ but not informed awareness domain $(\mathrm{r}=.073, \mathrm{P}=.331)($ Table 3). The reliability of the 2 -factor self-care maintenance structure was high (FDS=0.87).

\section{Self-Care Management Scale}

\section{Factor Analysis, Convergent Validity, and Reliability}

A 2-factor multidimensional structure to self-care management had a marginal fit $(\chi 2=26.7, \mathrm{p}=0.004 ; \mathrm{RMSEA}=0.093 ; \mathrm{CFI}=0.900, \mathrm{TLI}=0.886, \mathrm{SRMR}=0.059)$ (Figure 1 and Table 2). One self-care management factor was named "early recognition and response" with 3 items (i.e., \#12. recognizing symptoms of heart disease, \#13 reducing activity, and \#17 evaluating treatment effectiveness). A second factor was named "delayed response" with 3 items (i.e. \#14 taking nitroglycerine, \#15 calling the doctor, and \#16 taking an aspirin). Note that item \#11 is a dichotomous question regarding the presence of symptoms in the prior month. It is not used in scoring but instead used only to select those who were symptomatic and therefore eligible to complete the self-care management scale. Item-test correlations for the self-care management scale ranged from .55 to .66 with no redundancy or low correlation was found. The item with the lowest item-test correlation was item \# 15 ( $\mathrm{r}=.55)$, calling the doctor.

Convergent validity showed that both self-care management factors were associated significantly with adherence (early recognition and response $\mathrm{r}=.217, \mathrm{P}=.003$; delayed response $\mathrm{r}=.211, \mathrm{P}<.001$ ) but neither was associated significantly with decision-making (Table 3 ). The reliability of the 2-factor self-care management structure was sufficient (FDS=0.76). 


\section{Self-Care Confidence Scale}

\section{Factor Analysis, Convergent Validity, and Reliability}

Item-test correlations were all sufficiently high. A unidimensional factor structure of selfcare management items had good fit $\left(\chi^{2}=35.5, \mathrm{p}<0.001 ; \mathrm{RMSEA}=0.080 ; \mathrm{CFI}=0.966, \mathrm{TLI}=0.944\right.$, $\mathrm{SRMR}=0.031)$ (Figure 1 and Table 2). Item-test correlations for this scale ranged from .61 to .81 .

Self-care confidence was associated significantly with both adherence $(\mathrm{P}<.001)$ and the DCMI (total score, informed awareness and self-appraisal domains; $\mathrm{P}<.001$ ) (Table 3 ). The reliability of the unidimensional self-care confidence structure was good $(\alpha=0.84)$.

\section{Discussion}

The purpose of this study was to test the psychometric properties of a new measure of self-care designed for use with individuals with coronary heart disease, the SC-CHDI. The results of this study provide evidence that the SC-CHDI is sufficiently valid and reliable to encourage further testing.

These findings support the conceptual basis of self-care in patients with CHD as a process of maintenance that includes both consultative and autonomous behaviors and management, with symptom awareness and response. In this analysis the self-care maintenance "consultative behaviors" reflect those prescribed by healthcare providers because they provide secondary prevention benefits (Fraker et al., 2007). The four self-care maintenance "autonomous behaviors" are related to lifestyle modification and risk reduction. These autonomous behaviors and require self-motivation and adaption of daily behaviors (Slovinec D'Angelo, Pelletier, Reid, \& Huta, 2014).

Although the fit statistics were only adequate for the two-dimension model of self-care maintenance, conceptually, these two domains are consistent with the middle range theory of 
self-care of chronic illness (Riegel et al., 2012).The self-care maintenance behaviors are performed to maintain physical and emotional stability and preserve health. Riegel et al (2012) described self-care maintenance in chronic illness as comprised of adherence behaviors to maintain stability. They further differentiated the behaviors as either activities directed or influenced by others (e.g. healthcare providers) and then "agreed" upon by the individual; or adopted by the individual based upon personal health goals. Our results support a similar 2-factor structure of self-care maintenance for patients with CHD that consists of consultative behaviors that are influenced by others and autonomous behaviors that require self-motivation.

Considering the SC-CHDI management scale, the fit statistics were adequate for the twodimension model with two factors identified: "early recognition and response" and "delayed response". These factors are consistent with the theoretical underpinnings of self-care as a naturalistic decision making process that involves both symptom monitoring and symptom management. Logically, patients need to recognize and appropriately label their symptoms as related to CHD before they can be expected to respond appropriately. Once labeled, an early response is desired. However, a large body of literature illustrates that a delayed response to CHD symptoms is extremely common (DeVon, Burke, Nelson, Zerwic, \& Riley, 2014;

Lichtman et al., 2015; Wechkunanukul et al., 2016). A plethora of interventions have been tested aimed at reducing prehospital delay. However, it is possible that delays in seeking treatment are at least partially due to failure to adequately recognize and label CHD symptoms. Use of the SCCHDI in research will elucidate how well CHD patients recognize and label their symptoms and how they respond to those symptoms. This knowledge may help clinicians and researchers to develop better interventions to decrease treatment seeking delay in the future.

The SC-CHDI confidence scale is a promising measure of self-care self-efficacy that may 
help identify targets for future interventions. We have previously shown in populations with heart failure that confidence moderates the relationship between self-care and outcomes and is important to consistent engagement in self-care (Riegel et al., 2011). We have also demonstrated that self-care confidence reflects self-efficacy (Dickson, Deatrick, \& Riegel, 2008) and is essential to developing the skills needed for adequate self-care (Dickson et al., 2014). Since improving self-efficacy is central to many interventions targeting behaviors like diet adherence, exercise and symptom monitoring (Katch \& Mead, 2010; Lorig \& Holman, 2003), the self-care confidence scale is anticipated to be useful as a measure of self-care self-efficacy that can direct individualized counseling.

In validity testing the self-care maintenance scale was most highly correlated with the MOS General Adherence Survey. These correlations were anticipated because the behaviors included in the maintenance scale are those recommended to maintain health and stability. The self-care maintenance was also correlated with decision making, as anticipated, because self-care is said to be a decision-making process. We were surprised, however, that none of the decision making scores were correlated with self-care management, which is said to reflect a process of making decisions about how to treat symptoms when they occur. It may be that these patients were not being reflective in the decision-making process, an issue discussed at some length in the theory of self-care of chronic illness. Or, perhaps they do not have adequate information. Or, if they have the necessary information, perhaps they are critically evaluating their optionsdiscussed as reflection in the theory. Without a qualitative component to this study, we remain unclear why self-care management was unrelated to decision making.

Limitations to this analysis include the predominately Caucasian sample who responded to the survey. Additional testing is needed in diverse populations. Efforts are currently underway 
to translate the instrument into Spanish and Italian for additional psychometric testing. Another limitation of the study was that we did not have access to clinical data to confirm CHD in the entire sample. Test-retest reliability testing is also needed. A strength of the study was that the sample size was drawn from numerous areas of the United States.

\section{Conclusions}

Self-care is an essential component in the clinical management of patients with CHD. Improving self-care has important implications, especially with the increasing number of individuals living with CHD, now estimated at 15.5 million (Mozaffarian et al., 2016). Our results suggest that the SC-CHDI may fill an important gap in existing research. Although additional psychometric testing is needed, the SC-CHDI is anticipated to be useful in research aimed at understanding and improving self-care among patients with CHD. 


\section{Figure legend}

\section{Figure 1: Self-Care of Coronary Heart Disease Inventory.}

A multidimensional self-care maintenance factor $(\chi 2=124.6, \mathrm{p}<0.001$; $\mathrm{RMSEA}=0.088$;

$\mathrm{CFI}=0.904, \mathrm{TLI}=0.833, \mathrm{SRMR}=0.065)$ was identified that captured patients' "consultative behaviors" and "autonomous behaviors". A multidimensional self-care management factor $(\chi 2=26.7, p=0.004 ; \mathrm{RMSEA}=0.093 ; \mathrm{CFI}=0.900, \mathrm{TLI}=0.886, \mathrm{SRMR}=0.059)$ was identified that captured patients" "early recognition and response" and "delayed response". A unidimensional self-care confidence factor $\left(\chi^{2}=35.5, \mathrm{p}<0.001 ; \mathrm{RMSEA}=0.080 ; \mathrm{CFI}=0.966, \mathrm{TLI}=0.944\right.$, SRMR=0.031) was identified that captured patients' self-efficacy in following treatment advice, and in recognizing, evaluating and treating symptoms. 


\section{References}

Brown, T. (2003). Confirmatory factor analysis of the Penn State Worry Questionnaire: Multiple factors or method effects? . Behaviour Research and Therapy, 41, 1411-1426.

Buck, H. G., Dickson, V. V., Fida, R., Riegel, B., D'Agostino, F., Alvaro, R., \& Vellone, E. (2015). Predictors of hospitalization and quality of life in heart failure: A model of comorbidity, self-efficacy and self-care. International Journal of Nursing Studies 52(11), 1714-1722. doi: 10.1016/j.ijnurstu.2015.06.018

Chodosh, J., Morton, S. C., Mojica, W., Maglione, M., Suttorp, M. J., Hilton, L., . . Shekelle, P. (2005). Meta-analysis: chronic disease self-management programs for older adults. Annals of Internal Medicine, 143(6), 427-438.

DeVon, H. A., Burke, L. A., Nelson, H., Zerwic, J. J., \& Riley, B. (2014). Disparities in patients presenting to the emergency department with potential acute coronary syndrome: it matters if you are Black or White. Heart Lung, 43(4), 270-277. doi:

10.1016/j.hrtlng.2014.04.019

Dickson, V.V, Deatrick, J., \& Riegel, B. (2008). A typology of heart failure self-care management in non-elders. European Journal of Cardiovascular Nursing, 7(3), 171-181. doi: 10.1016/j.ejcnurse.2007.11.005.

Dickson, V., Melkus, G., Katz, S., Levine-Wong, A., Dillworth, J., Cleland, C., \& Riegel, B. (2014). Building Skill in Heart Failure Self-Care among Community Dwelling Older Adults: Results of a Pilot Study. Patient Education and Counseling, 96(2), 188-196. doi: http://dx.doi.org/10.1016/j.pec.2014.04.018 
Dickson, V., Nocella, J., Yoon, H.-W., Hammer, M., Melkus, G. D., . . Chyun, D. (2013). Cardiovascular Disease Self-Care Interventions. Nursing Research and Practice, 2013(407608). doi: 10.1155/2013/407608.

Eller, L. S., Lev, E. L., Yuan, C., \& Watkins, A. V. (2016). Describing Self-Care Self-Efficacy: Definition, Measurement, Outcomes, and Implications. International Journal of Nursing Knowledge. doi: 10.1111/2047-3095.12143

Flora, D., \& Curran, P. (2004). An empirical evaluation of alternative methods of estimation for confirmatory factor analysis with ordinal data. Psychological Methods, 9(466-491).

Fraker, T., Fihn, S., Gibbons, R., Abrams, J., Chatterjee, K., Daley, J., . . Yancy, C. (2007). 2007 Chronic Angina Focused Update of the ACC/AHA 2002 Guidelines for the Management of Patients With Chronic Stable Angina: A Report of the American College of Cardiology/American Heart Association Task Force on Practice Guidelines Writing Group to Develop the Focused Update of the 2002 Guidelines for the Management of Patients With Chronic Stable Angina. Circulation 116(23), 2762-2772.

Gaziano, T. (2007). Reducing the growing burden of cardiovascular disease in the developing world. Health Affairs (Millwood), 26(1), 13-24.

Gehi, A., Haas, D., Pipkin, S., \& Whooley, M. (2005). Depression and medication adherence in outpatients with coronary heart disease. Archives of Internal Medicine, 165, 2508-2513.

Gibbons, R., Abrams, J., Chatterjee, K., Daley, J., Deedwania, P., Douglas, J., . . Smith, S. (2003). ACC/AHA 2002 Guideline Update for the Management of Patients With Chronic Stable Angina--Summary Article: A Report of the American College of Cardiology/American Heart Association Task Force on Practice Guidelines (Committee 
on the Management of Patients With Chronic Stable Angina). Circulation 107(1), 149158.

Hays, R. D., Kravitz, R. L., Mazel, R. M., Sherbourne, C. D., DiMatteo, M. R., Rogers, W. H., \& Greenfield, S. (1994). The impact of patient adherence on health outcomes for patients with chronic disease in the Medical Outcomes Study. Journal of Behavioral Medicine, 17(4), 347-360.

Hisashige, A. (2013). The Effectiveness and Efficiency of Disease Management Programs for Patients with Chronic Diseases. Global Journal of Health Science, 5(2), 27-48. doi: 10.5539/gjhs.v5n2p27

Hu, L., \& Bentler, P. (1999). Cutoff criteria for fit indexes in covariance structure analysis: Conventional criteria versus new alternatives. Structural Equation Modeling, 6(1), 1-55.

Huffman, J. C., Moore, S. V., DuBois, C. M., Mastromauro, C. A., Suarez, L., \& Park, E. R. (2015). An exploratory mixed methods analysis of adherence predictors following acute coronary syndrome. Psychology, Health \& Medicine, 20(5), 541-550. doi: $10.1080 / 13548506.2014 .989531$

Katch, H., \& Mead, H. (2010). The role of self-efficacy in cardiovascular disease selfmanagement: a review of effective programs. Patient Intelligence, 2(33-44).

Lichtman, J. H., Leifheit-Limson, E. C., Watanabe, E., Allen, N. B., Garavalia, B., Garavalia, L. S., . . Curry, L. A. (2015). Symptom recognition and healthcare experiences of young women with acute myocardial infarction. Circulation: Cardiovascular Quality Outcomes, 8(2 Suppl 1), S31-38. doi: 10.1161/circoutcomes.114.001612

Lorig, K. R., \& Holman, H. (2003). Self-management education: history, definition, outcomes, and mechanisms. Annals of Behavioral Medicine, 26(1), 1-7. 
Lynn, M. (1986). Determination and quantification of content validity. Nursing Research, 35(6), $382-385$.

Maddox, T. M., \& Ho, P. M. (2009). Medication adherence and the patient with coronary artery disease: challenges for the practitioner. Current Opinions in Cardioliology, 24(5), 468472. doi: 10.1097/HCO.0b013e32832ed62d.

McDowell, I. (2006). The theoretical and technical foundations of health measurement Measuring Health: A Guide to Rating Scales and Questionnaires (3rd ed.). New York: Oxford Unversity Press.

Miller, D., \& Byrnes, J. (2001). Adolescents' decision making in social situtions: A selfregulation perspective. Applied Developmental Psychology, 22, 237-256.

Moser, D., Kimble, L., Alberts, M., Alonzo, A., Croft, J., Dracup, K., . . Zerwic, J. (2006). Reducing delay in seeking treatment by patients with acute coronary syndrome and stroke: A scientific statement from the American heart Association Council on Cardiovascular Nursing and Stroke Council. Circulation, 114, 168-182.

Mozaffarian, D., Benjamin, E. J., Go, A. S., Arnett, D. K., Blaha, M. J., Cushman, M., . . . Turner, M. B. (2016). Heart Disease and Stroke Statistics-2016 Update: A Report From the American Heart Association. Circulation, 133(4), e38-e360. doi: 10.1161/cir.0000000000000350

Polit, D., \& Beck, C. (2006). The content validity index: are you sure you know what's being reported? Critique and recommendations. Research in Nursing \& Health, 29(5), 489497. doi: 10.1002/nur.20147

Polit, D., Beck, C., \& Owen, S. (2007). Is the CVI an acceptable indicator of content validity? Appraisal and recommendations. Research in Nursing \& Health, 30(4), 459-467. 
Riegel, B., Jaarsma, T., \& Stromberg, A. (2012). A middle-range theory of self-care of chronic illness. ANS Advances in Nursing Science, 35(3), 194-204. doi: 10.1097/ANS.0b013e318261b1ba.

Riegel, B., Lee, C., Albert, N., Lennie, T., Chung, M., Song, E., . . Moser, D. (2011). From novice to expert: confidence and activity status determine heart failure self-care performance. Nursing Research, 60(2), 132-138.

Schnermelleh-Engel, K., Moosbrugger, H., \& Muller, H. (2003). Evaluating the fit of structural eqaution models: tests of significance and descriptive goodness-of-fit measures. Methods of Psychological Research Online, 8(2), 23-74.

Slovinec D'Angelo, M. E., Pelletier, L. G., Reid, R. D., \& Huta, V. (2014). The roles of selfefficacy and motivation in the prediction of short- and long-term adherence to exercise among patients with coronary heart disease. Health Psychology, 33(11), 1344-1353. doi: $10.1037 /$ hea0000094

Smith, S. C., Benjamin, E. J., Bonow, R. O., Braun, L. T., Creager, M. A., Franklin, B. A., . . Taubert, K. A. (2011). AHA/ACCF Secondary Prevention and Risk Reduction Therapy for Patients With Coronary and Other Atherosclerotic Vascular Disease: 2011 Update: A Guideline From the American Heart Association and American College of Cardiology Foundation. Circulation, 124(22), 2458-2473. doi: 10.1161/CIR.0b013e318235eb4d

Tabachnick, B., \& Fidell, L. (2013). Using Multivariate Statistics (6th ed.). Boston: Allyn \& Bacon.

Toft, U., Kristoffersen, L., Aadahl, M., von Huth Smith, L., Pisinger, C., \& Jorgensen, T. (2007). Diet and exercise intervention in a general population mediators of participation and adherence: the Inter99 study. European Journal of Public Health $\backslash$, 17(5), 455-463. 
Vellone, E., Fida, R., D'Agostino, F., Mottola, A., Juarez-Vela, R., Alvaro, R., \& Riegel, B. (2015). Self-care confidence may be the key: A cross-sectional study on the association between cognition and self-care behaviors in adults with heart failure. Int J Nurs Stud, 52(11), 1705-1713. doi: 10.1016/j.ijnurstu.2015.06.013

Wechkunanukul, K., Grantham, H., \& Clark, R. A. (2016). Global review of delay time in seeking medical care for chest pain: An integrative literature review. Aust Crit Care. doi: 10.1016/j.aucc.2016.04.002

Wu, J., Moser, D., Chung, M., \& Lennie, T. (2008). Objectively measured, but not self-reported, medication adherence independently predicts event-free survival in patients with heart failure. J Card Fail, 14(3), 203-210.

Yu, C. (2002). Evaluating Cutoff Criteria of Model Fit Indices for Latent Variable Models with Binary and Continuous Outcome. Doctoral dissertation, University of California, Los Angeles. 